\title{
Macroscopic strains in granular materials accounting for grain rotations
}

\author{
N.P. Kruyt • O. Millet • F. Nicot
}

Received: date / Accepted: date

\begin{abstract}
Granular materials are special materials, from the continuum-mechanical viewpoint, in the sense that they possess a clear microstructure of grains and intergrain contacts. In addition, the grains have translational as well as rotational degrees of freedom.

Here a micromechanical expression is formulated for the average displacement gradient tensor in terms of the grain displacements and rotations for the two-dimensional case. It is based on a tessellation of the granular assembly into closed loops, along whose boundary the displacement field is defined in terms of the grain displacements and rotations. An important consideration for this expression is that it must satisfy the surfaceadditivity property, according to which the average displacement gradient of a combined two-dimensional surface is determined by the average displacement gradients of the constituent surfaces (and weighted by their areas).
\end{abstract}

This study is an extended version of that presented by the authors, Deformation analysis of granular materials at micro and macro scales, at International Symposium on Geomechanics from Micro to Macro, Cambridge, United Kingdom (in press)

\section{N.P. Kruyt}

Department of Mechanical Engineering

University of Twente, The Netherlands

E-mail: n.p.kruyt@utwente.nl

O. Millet

LaSIE-UMR CNRS 7356

La Rochelle University, France

E-mail: olivier.millet@univ-lr.fr

F. Nicot

Irstea Grenoble, France

E-mail: francois.nicot@irstea.fr
The developed micromechanical expression is verified by comparing its results with the macroscopic deformation as determined from the displacements at the boundary. Results of DEM simulations of a biaxial test (where the average rotation is equal to zero) and a shear test (where the average rotation is not equal to zero) are employed for this verification. The developed micromechanical expression for the displacement gradient is subsequently used to study deformation patterns in a complex case of a biaxial test where a shear band is formed.

Keywords Granular materials · Displacement gradient tensor · Micromechanics · Grain displacements and rotations

\section{Introduction}

For several decades, mechanicians and physicists have been fascinated by the properties of granular materials $[11,17,29,33]$ : are they fluid-like materials or solidlike materials? According to the applied loading and to the porosity of the specimen (i.e. the ratio between void volume and total volume) at hand, different types of behaviour may be observed. Granular materials can flow like a fluid (as in grain avalanches $[2,3,31,34]$ ), or exhibit mechanical strength as solids do $[4,6,8,28]$. In fact, an important characteristic of granular materials is that they possess an internal structure, determined by the constituent grains and their arrangement. Grains can be regarded as the elementary scale of granular materials, except when grains can be deformed in the same range of magnitude as the whole specimen.

The prediction of macroscopic strains in a given granular volume is of great interest, both for scientific and engineering purposes. Most soils are granular 
geomaterials, whose strains have to be correctly modelled over a given loading path, for instance in order to check their compatibility with a given civil engineering project. The investigation of the stability of natural slopes also requires that the strain field within the soil body is correctly determined. A related challenge concerns the development of powerful constitutive models for granular materials. This is an important field in engineering sciences, in order to perform numerical simulations to predict the response of a given structure under different loading conditions. Even though considerable progress has been achieved in this domain, further research studies are still expected to better relate the macroscopic properties of the materials to their inherent microstructure.

The basic properties of granular materials are deeply related to the specific kinematics of the grains. Particular patterns of grains in contact may be present. These patterns evolve over a given loading path, due to the fact that grains in contact may slide or may rotate, possibly leading to the opening of the contact (or disruption of the contact). Alternatively, additional contacts may be created due to grains moving towards one another. Basically, two kinds of patterns exist within a granular assembly. Firstly, quasi-linear patterns of grains (also denoted as force chains) that are formed by a few grains in contact that are more or less aligned $[23,32,33]$. Such patterns account for the transmission of significant stress levels within the material. Secondly, grains arrange in closed loops (in two-dimensional conditions) or in some sort of aggregates (in three-dimensional conditions), consisting of a collection of grains in contact $[24,25,35,36,37]$. Such arrangements account for the deformation of the specimen, especially when grains are nearly rigid. Basically, the deformation stems from the relative displacements between grains (by sliding or rolling). As a result, an outstanding feature of granular materials is that stress properties relate to the columnlike patterns of grains, whereas strain properties result from the deformability of aggregates (or closed loops in two-dimensional conditions), i.e. the deformation of the void space. Further research is still required to thoroughly understand the complex interrelations between these two patterns (column-like grain patterns and aggregates), even though interesting investigations have already been performed $[35,37]$.

Granular materials are special materials, from the continuum-mechanical viewpoint, in that they possess a distinct discrete character where the constituent grains have translational as well as rotational degrees of freedom. Thus, granular materials could be considered as micropolar materials (see for example [16]).
In the context of multi-scale approaches applied to granular media, expressions for the average strain tensor as a function of microscopic quantities have been studied by many authors.

Such expressions relating characteristics of grains to macroscopic, continuum characteristics are valuable in interpreting experimental data or results from numerical simulations, or for micromechanical studies. Micromechanical expressions are preferably expressed in terms of deformation characteristics at the level of grains, i.e. involving relative displacements. In the present study, this micromechanical approach is adopted.

A variety of expressions for the average strain tensor has been developed and compared by performing numerical simulations, see for instance $[4,5,6,7,12,14$, $15,21,24,25,26,27,30]$. A critical appraisal of many of these expressions is given in $[5,14]$. However, few attempts have been made so far to account for the influence of grain rotations on the average deformation gradient tensor. For instance, in [30] the strain tensor is computed by interpolation using a fixed grid. The granular assembly is discretized in a set of subvolumes containing a sufficiently large number of grains. The movements of the grains, including both translational and rotational components, are computed at each node of the grid within each subvolume. When the node is lcoated inside a grain, the exact displacement can be determined from the solid rigid motion of the grain. When the node belongs to the pore space, the movement is approximated by interpolation according to the location of this node with respect to the neighboring grains. Using a different line of reasoning, in [6] a description has recently been given of the deformation gradient tensor for loose two-dimensional granular assemblies that also accounts for the grain rotations. This approach involves the motion of the void to completely formulate the average deformation gradient of the granular medium. For the selected Representative Elementary Volume (REV for short), the surface-additivity property (see Section 2 ) for the average displacement gradient tensor is not easily verified.

The objective of this study is to formulate measures of average deformation at the macro-scale, continuummechanical level, in terms of the kinematical variables, grain displacements and grain rotations, at the microscale level of grains and intergrain contacts for dense granular assemblies.

The outline of this study is as follows. Firstly, the average-strain theorem is recalled in Section 2. Then the basics of micromechanics of granular materials are summarised in Section 3. The proposed expression for the average displacement gradient tensor, in terms of the translations and rotations of the grains (as well as 
some additional geometrical characteristics), is given in Section 4. This is followed by a verification of the developed micromechanical expression in Section 5. Employing this expression, the deformation in a complex case that involves a shear band is analysed in Section 6.

\section{Average-strain theorem}

Here the average-strain theorem is recalled, and its consequence for the micromechanical expression for the average strain is discussed. The continuum-mechanical displacement field is denoted by $\mathbf{u}(\mathbf{x})$, where $\mathbf{x}$ is the position vector. The (small-deformation) displacement gradient tensor $\boldsymbol{\alpha}$ is defined by

$\alpha_{i j}=\frac{\partial u_{i}}{\partial x_{j}}$.

In many cases the average displacement gradient tensor $<\alpha_{i j}>_{S}$ for a two-dimensional surface $S$ is of interest. It is given by

$<\alpha_{i j}>_{S}=\frac{1}{A} \int_{S} \frac{\partial u_{i}}{\partial x_{j}} d S$,

where $A$ is the area of surface $S$. Using Gauss' theorem, this equation can be rewritten as

$<\alpha_{i j}>_{S}=\frac{1}{A} \int_{B} u_{i} n_{j} d s$,

where $B$ is the boundary of the surface $S, \mathbf{n}$ is the outward unit normal vector and $d s$ is the arc-length element along the boundary, see also Figure 1. This important result is the average-strain theorem (see for example [1]), according to which the average displacement gradient tensor is completely determined by the displacement at the boundary (and the geometry of the boundary).

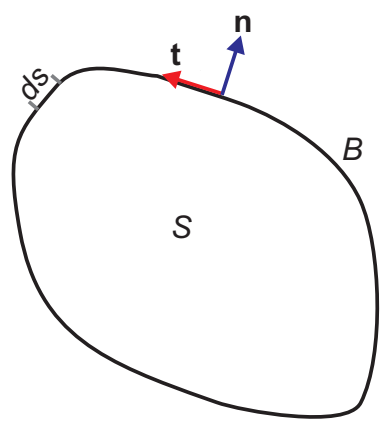

Fig. 1: Two-dimensional area $S$, boundary $B$ and normal vector $\mathbf{n}$ and tangential vector $\mathbf{t}$. The arc-length element along the boundary is denoted by $d s$.
A consequence of this definition is that the average displacement gradient tensor should be given by an expression that is surface-additive (in the two-dimensional case considered here, rather than volume-additive in the three-dimensional case). When the surface $S$ consists of two disjoint surfaces $S_{1}$ and $S_{2}$, with corresponding average displacement gradient tensors $<\boldsymbol{\alpha}>_{S_{1}}$ and $<\boldsymbol{\alpha}>_{S_{2}}$ and areas $A_{1}$ and $A_{2}$ respectively, then the following equality should be satisfied

$$
\begin{aligned}
A<\alpha_{i j}>_{S} & =\int_{S} \frac{\partial u_{i}}{\partial x_{j}} d S=\int_{S_{1}} \frac{\partial u_{i}}{\partial x_{j}} d S+\int_{S_{2}} \frac{\partial u_{i}}{\partial x_{j}} d S \\
& =A_{1}<\alpha_{i j}>_{S_{1}}+A_{2}<\alpha_{i j}>_{S_{2}}
\end{aligned}
$$

provided that there is no discontinuity in the displacement at the interface between the two surfaces. Note that this important additivity property is also satisfied by the definition of the average stress tensor ${ }^{1}$ proposed in $[13,18,19]$.

The displacement at the boundary will in general consist of two parts, one due to grain displacements and one due to grain rotations. Here an order of magnitude analysis of these contributions is first performed. The displacement due to grain translations at the boundary will scale as $\epsilon L$, where $\epsilon$ is a measure of the imposed strain and $L$ is a macroscopic length scale. The displacement at the boundary due to grain rotation will scale as $\omega R$, where $\omega$ is a measure of the grain rotation and $R$ is an average grain radius. For granular materials the scale for the grain rotations $\omega$ equals $\epsilon$. Hence, the ratio of the contribution of grain rotations over the contribution of grain translations to the displacement at the boundary scales as $R / L$ (see also [6]). This means that the contribution of grain rotations is small for very large assemblies. The contribution of grain rotations may possibly be important in small subsystems or in systems with large gradients in displacements, such as in shear bands.

The Einstein summation convention is employed here, in which a summation is implied over repeated coordinate subscripts.

\section{Micromechanics}

Since grains in granular assemblies are generally very stiff, the interaction between grains can be considered as taking place at contact points, or contact regions of very small size.

1 This definition involves not only contact forces between grains, but also inertia forces in dynamical situations (see also [20] for the associated DEM simulations). 
Kruyt, N.P. ; Millet, O. ; Nicot, F. (2014) Macroscopic strains in granular materials accounting for grain rotations. Granular Matter, vol. 16, $n^{\circ}$ 6, p. 933-944. DOI 10.1007/s10035-014-0523-3

The position vector of the centre of grain $p$ is denoted by $\mathbf{X}^{p}$. The position vector of the contact point between grains $p$ and $q$ is given by $\mathbf{X}^{C(p q)}$. The vector from the centre of grain $p$ to the contact point between grains $p$ and $q$ is $\mathbf{R}^{p q}$. Hence $\mathbf{R}^{p q}=\mathbf{X}^{C(p q)}-\mathbf{X}^{p}$ and $\mathbf{R}^{q p}=\mathbf{X}^{C(q p)}-\mathbf{X}^{q}\left(\right.$ with $\left.\mathbf{X}^{C(q p)}=\mathbf{X}^{C(p q)}\right)$. These vectors are shown in Figure 2.

The kinematical degrees of freedom of grain $p$ are its displacement vector $\mathbf{U}^{p}$ and its rotation $\omega^{p}$. In the two-dimensional case considered here, this rotation is a scalar quantity. As grains are stiff, the displacement inside grains closely approximates that of rigid body motion

$\mathbf{u}(\mathbf{x})=\mathbf{U}^{p}+\boldsymbol{\Omega}^{p} \wedge\left(\mathbf{x}-\mathbf{X}^{p}\right)$.

where $\boldsymbol{\Omega}^{p}$ denotes the rotation vector of grain $p$. For two-dimensional granular assemblies, $\boldsymbol{\Omega}^{p}=\omega^{p} \mathbf{e}_{\mathbf{3}}$ where $\mathbf{e}_{\mathbf{3}}$ is the unit vector normal to the two-dimensional plane considered.

The relative displacement $\Delta^{p q}$ at the contact point between grains $p$ and $q$ is then given by

$\Delta^{p q}=\left[\mathbf{U}^{p}-\mathbf{U}^{q}\right]+\left[\boldsymbol{\Omega}^{p} \wedge \mathbf{R}^{p q}-\boldsymbol{\Omega}^{q} \wedge \mathbf{R}^{q p}\right]$.

The relative displacement consists of two contributions, the first term that involves the grain translations and the second term that involves grain rotations. The relative displacements are important for the constitutive relation at the contact level that links (increments of) relative displacements to (increments of) contact forces.

For future conciseness in notation, the vector $\hat{\mathbf{R}}$, that is associated with the vector $\mathbf{R}$, is defined by

$\hat{\mathbf{R}}=-\mathbf{E} \cdot \mathbf{R}$,

where $E_{i j}$ denotes the two-dimensional permutation symbol. The vector $\hat{\mathbf{R}}$ is obtained from the vector $\mathbf{R}$ by

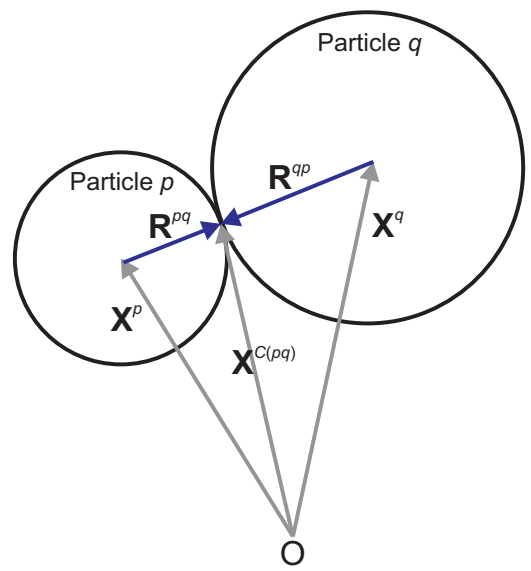

Fig. 2: Contact geometry and position vectors. counter-clockwise rotation over $90^{\circ}$. Terms for the displacement due to grain rotations can now be expressed compactly as $\boldsymbol{\Omega} \wedge \mathbf{R}=\omega \hat{\mathbf{R}}$.

\section{Expression for average displacement gradient, incorporating grain rotations}

The average deformation of the assembly can be described in terms involving the grain kinematics. In this Section, basic results described in $[6,24]$ are reviewed and extended. The differences and commonalities between these results and the current results are discussed at the end of this Section.

In order to evaluate the average displacement gradient tensor in Eq. (3), it is necessary to specify the boundary $B$, i.e. the surface $S$ under consideration, and the displacement vector $\mathbf{u}$ at the boundary $B$.

Granular assemblies consist of grains and voids. Inside grains, the displacement field can be expressed in terms of the displacement of the grain centres $\mathbf{U}^{p}$ and the grain rotations $\omega^{p}$, see Eq. (5). To avoid the complexities involved in the definition of a displacement field inside the voids (see [6]), here it is preferred to select the boundary $B$ such that it is always part of the grains. This is possible in the two-dimensional case considered here when the granular assembly is dense.

Loose granular assemblies have also been considered in [6]. Then the void phase has to be taken into account, and the continuation of the displacement field into the void phase must be defined, Additionally, the definition of the boundary of the REV is not so straightforward as for dense granular assemblies.

In the dense case the network of intergrain contacts tessellates the surface occupied by the two-dimensional assembly into closed loops (or polygons, or cycles in the

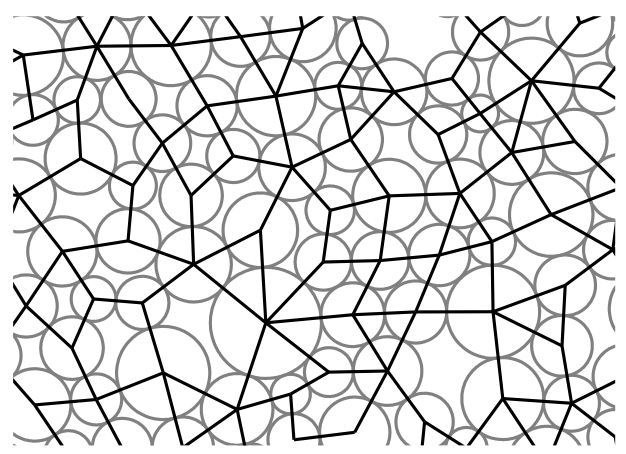

Fig. 3: Tessellation of part of an assembly: grains, intergrain contacts (indicated by lines joining the centres of grains in contact) and loops that are formed by contacts. 


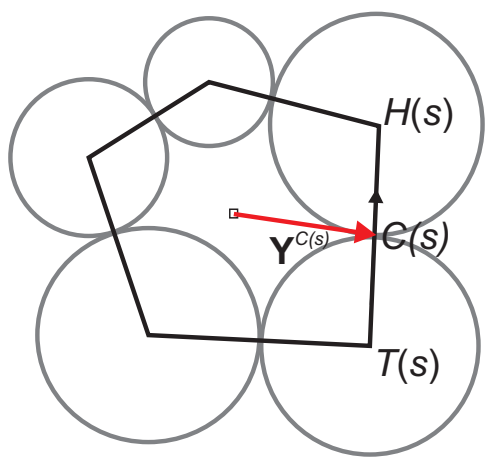

Fig. 4: Single loop, with 'head' $H(s)$, 'tail' $T(s)$ and contact point $C(s)$ of directed side $s$. Also shown is the vector $\mathbf{Y}^{C(s)}$ from the centre of the loop to the contact point corresponding to side $s$.

terminology of graph theory) of intergrain contacts [24, 25], as illustrated in Figure 3.

To evaluate the integral in Eq. (3), a single loop is first considered. Then, when considering a larger surface (or an REV), the surface-additivity property Eq. (4) can be used. Therefore, only the definition of the average displacement gradient for a single loop is necessary.

To simplify the notation in the following derivations, the integral $\int_{B} u_{i} t_{j} d s$ involving the tangential vector $\mathbf{t}$, which is indicated in Figure 1, (rather than the normal vector $\mathbf{n}$ ) is considered. Since $\mathbf{n}=\mathbf{E} \cdot \mathbf{t}$, the integrals are related by

$\int_{B} u_{i} n_{j} d s=E_{j k} \int_{B} u_{i} t_{k} d s$.

The integral over the boundary of a loop is split into parts that correspond to the sides $s$ of the loop, where each side is from the centre of a grain to the centre of the next grain, as illustrated in Figure 4:

$\int_{B} u_{i} t_{k} d s=\sum_{s} \int_{B^{s}} u_{i} t_{k} d s$.

Inside each grain the displacement varies linearly, see Eq. (5). The integral along a single, straight side $s$ can then be evaluated as

$$
\begin{aligned}
\int_{B^{s}} u_{i} t_{k} d s & =\frac{1}{2}\left[U_{i}^{H(s)}+U_{i}^{C(s)+}\right]\left[X_{k}^{H(s)}-X_{k}^{C(s)}\right] \\
& +\frac{1}{2}\left[U_{i}^{T(s)}+U_{i}^{C(s)-}\right]\left[X_{k}^{C(s)}-X_{k}^{T(s)}\right],
\end{aligned}
$$

where $\mathbf{U}^{H(s)}$ and $\mathbf{U}^{T(s)}$ are the displacements at the 'head' and the 'tail' of the directed side $s$ (see also Figure 4). The total displacements at the contact point are denoted by $\mathbf{U}^{C(s)+}$ and $\mathbf{U}^{C(s)-}$ (where $C(s)+$ is closest to $H(s)$ and $C(s)-$ is closest to $T(s))$. Note that these total displacements at the contact point are different for the two sides, as there is a relative displacement between the grains. The relative displacement $\Delta^{s}$ (defined in Eq. (6)) at the contact corresponding to side $s$ is therefore given by $\Delta^{s}=\mathbf{U}^{C(s)+}-\mathbf{U}^{C(s)-}$.

Using Eqs. (5) and (7) for the displacement inside grains, it follows that the total displacements at the contact point, $\mathbf{U}^{C(s)+}$ and $\mathbf{U}^{C(s)-}$, can be expressed in terms of grain displacements and rotations as

$$
\begin{aligned}
\mathbf{U}^{C(s)+} & =\mathbf{U}^{H(s)}+\omega^{H(s)} \hat{\mathbf{R}}^{H(s) C(s)}, \\
\mathbf{U}^{C(s)-} & =\mathbf{U}^{T(s)}+\omega^{T(s)} \hat{\mathbf{R}}^{T(s) C(s)} .
\end{aligned}
$$

Note that $\mathbf{R}^{H(s) C(s)}=\mathbf{X}^{C(s)}-\mathbf{X}^{H(s)}$ and $\mathbf{R}^{T(s) C(s)}=$ $\mathbf{X}^{C(s)}-\mathbf{X}^{T(s)}$. Using Eq. (11), Eq. (10) can be rewritten as

$\int_{B^{s}} u_{i} t_{k} d s=\left[U_{i}^{H(s)} X_{k}^{H(s)}-U_{i}^{T(s)} X_{k}^{T(s)}\right]$

$-\left[U_{i}^{H(s)}-U_{i}^{T(s)}\right] X_{k}^{C(s)}$

$-\frac{1}{2}\left[\omega^{H(s)} \hat{R}_{i}^{H(s) C(s)} R_{k}^{H(s) C(s)}-\omega^{T(s)} \hat{R}_{i}^{T(s) C(s)} R_{k}^{T(s) C(s)}\right]$.

The following auxiliary relation will be used in the following

$\sum_{s}\left(\phi^{H(s)}-\phi^{T(s)}\right)=0$,

where $\phi$ is an arbitrary quantity that is associated with a grain. This relation is the discrete analogue of the relation $\oint_{C} \frac{d \phi}{d s} d s=0$, which is valid for closed contours C.

Since the first term in Eq. (12) cancels out due to the previous auxiliary relation (with $\phi=U_{i} X_{k}$ ), it follows that the average displacement gradient of a single loop $L$ (corresponding to the surface $S_{L}$ with area $A_{L}$ ) is given by

$$
\begin{aligned}
<\alpha_{i j}>_{S_{L}} & =\frac{1}{A_{L}} \sum_{s}\left[U_{i}^{H(s)}-U_{i}^{T(s)}\right] \hat{X}_{j}^{C(s)} \\
& +\frac{1}{2}\left[\omega^{H(s)} \hat{R}_{i}^{H(s) C(s)} \hat{R}_{j}^{H(s) C(s)}\right. \\
& \left.-\omega^{T(s)} \hat{R}_{i}^{T(s) C(s)} \hat{R}_{j}^{T(s) C(s)}\right],
\end{aligned}
$$

where we have used the relation

$<\alpha_{i j}>_{S_{L}}=\frac{1}{A_{L}} \sum_{s} E_{j k} \int_{B^{s}} u_{i} t_{k} d s$, 
in combination with Eq. (12). Note also that (see also Eq. (7))

$$
\begin{aligned}
E_{j k} R_{k}^{H(s) C(s)} & =-\hat{R}_{j}^{H(s) C(s)}, \\
E_{j k} R_{k}^{T(s) C(s)} & =-\hat{R}_{j}^{T(s) C(s)},
\end{aligned}
$$

and

$$
E_{j k} X_{k}^{C(s)}=-\hat{X}_{j}^{C(s)} .
$$

Using the auxiliary relation Eq. (13) (with $\phi=X_{i}^{L 0}$, where $\mathbf{X}^{L 0}$ is the position vector of the centre of the loop $L$ ), the first term in Eq. (14) involving position vectors can be expressed in terms of position vectors relative to the centre of the loop

$$
\begin{aligned}
<\alpha_{i j}>_{L} & =\frac{1}{A_{L}} \sum_{s}\left\{\left[U_{i}^{H(s)}-U_{i}^{T(s)}\right] \hat{Y}_{j}^{C(s)}\right. \\
& +\frac{1}{2}\left[\omega^{H(s)} \hat{R}_{i}^{H(s) C(s)} \hat{R}_{j}^{H(s) C(s)}\right. \\
& \left.\left.-\omega^{T(s)} \hat{R}_{i}^{T(s) C(s)} \hat{R}_{j}^{T(s) C(s)}\right]\right\}
\end{aligned}
$$

where the vector $\mathbf{Y}^{C(s)}=\mathbf{X}^{C(s)}-\mathbf{X}^{L 0}$ (see also Figure 4),

which reflects the local geometry. Note that the choice of position vector $\mathbf{X}^{L 0}$ is, in principle, arbitrary. The first term in this expression involves the contribution of displacements of grain centres to the average displacement gradient. This term was also obtained in [24, 25]. The current study extends the formulation in these studies by including the influence of grain rotations on the average displacement gradient, as in [6].

The formulated expression is such that it involves the difference of the displacement of the grains that are in contact, which forms a measure of deformation at the contact level (rather than the absolute grain displacement). The second term involves the contribution of grain rotations to the average displacement gradient.

Note that at a side shared by two loops, the displacement along the side is the same for these two loops. Hence, the displacement field implicit in the formulation is continuous. In Finite Element Method terminology, the loops can be considered as 'compatible' elements.

The average displacement gradient for the surface $S$ (with area $A$ ) of the full REV is now given by

$<\alpha_{i j}>_{S}=\frac{1}{A} \sum_{L} A_{L}<\alpha_{i j}>_{S_{L}}$,

ensuring that the surface-additivity property, Eq. (4), is always satisfied.

The expression for the average-displacement gradient tensor is formulated in terms of the displacements and rotations of the grains. By basing the formulation on elementary loops, the surface-additivity property, Eq. (4), is always satisfied, contrary to many micromechanical expressions for the strain tensor $[7,9,12]$. The final expression is such that it involves the difference of displacements of grains that are in contact. Regarding the contribution of the grain displacements, the current expression is equivalent to that given in [24]. In the current formulation the contribution of the grain rotations is also accounted for. In [6], grain rotations have already been taken into account, but the micromechanical expression is given in terms of grain displacements (so not in terms of relative displacements that form a measure of deformation at the contact level). In addition, the formulation in [6] does not directly satisfy the surfaceadditivity property, Eq. (4). Thus, the current formulation forms a synthesis of formulations given in $[6,24]$. In the following, extensive verification of the current formulation is described.

\section{Verification by DEM simulations}

To verify the developed micromechanical expression, detailed information is required on the grain displacements and rotations. This information is conveniently obtained from DEM simulations [10]. DEM simulations have been performed of a biaxial test (see Section 5.1) and of a shear test (see Section 5.2).

The grain interaction model employed here in the DEM simulations is represented by two linear springs in directions normal and tangential to the contact, with corresponding normal and tangential spring stiffnesses $k_{n}$ and $k_{t}$, respectively, in combination with a Coulomb friction law, $\left|f_{t}^{c}\right| \leq \mu f_{n}^{c}$, where $f_{n}^{c}$ and $f_{t}^{c}$ are the normal and the tangential component of the force vector at contact $c$ and $\mu$ is the interparticle friction coefficient. Here the value of $\mu=0.5$ has been selected. Since the grains are generally stiff, the deformation (or "overlap") of grains will be small. This is ensured here by selecting the normal stiffness $k_{n}$ such that $p_{0} / k_{n}=10^{-3}$ (this is a dimensionless quantity in the two-dimensional case that is considered here), where $p_{0}$ is the initial hydrostatic confining pressure. The tangential stiffness $k_{t}$ is selected such that $k_{t} / k_{n}=0.5$.

An initial assembliy consisting of 50000 disks has been generated from a log-normal distribution for the grain radius, where the ratio of the standard deviation over the mean grain radius equals 0.25 . The initial assembly has been created by: (1) generating an assembly where the grains are not in contact, and (2) subsequently compressing the assembly slowly, using a small value for the interparticle friction coeffcient, until the 
required isotropic stress state is attained. The initial solid fraction of the sample was 0.832 .

Major and minor principal stresses are denoted by $\sigma_{1}$ and $\sigma_{2}$, respectively. Invariants $p$ and $q$ of the stress tensor are given by $q=\left(\sigma_{1}-\sigma_{2}\right) / 2$ and the mean pressure $p=\left(\sigma_{1}+\sigma_{2}\right) / 2$. Major and minor principal strains are denoted by $\epsilon_{1}$ and $\epsilon_{2}$, respectively. Volumetric strain $\epsilon_{V}$ is given by $\epsilon_{V}=\epsilon_{1}+\epsilon_{2}$.

\subsection{Biaxial test}

As a verification of the micromechanical expression for the average displacement gradient, the results of DEM simulation of a biaxial test have been used to compare the average displacement gradient according to Eqs. (16) and (17) with the macroscopic displacement gradient that is determined from the deformation of the periodic box.

In the biaxial test the principal strain $\epsilon_{1}$ has been imposed, while the lateral minor principal stress $\sigma_{2}$ is kept constant. Periodic boundaries have been employed in order to obtain largely homogeneous deformation patterns.

The macroscopic response is shown in Figure 5 for the relative shear strength $q / p$ and the volumetric strain $\epsilon_{V}$. The results show behaviour that is typical for an initially dense granular assembly. The shear strength $q / p$ rapidly increases to a peak value, after which some softening occurs. Note that through the use of periodic boundaries the formation of large-scale, persistent shear bands is suppressed. The volumetric strain $\epsilon_{V}$ shows a small initial compression, followed by dilation until a steady state is attained at large imposed strain $\epsilon_{1}$ in which deformation occurs at constant stress and solid fraction.

For the micromechanical expression, Eqs. (16) and (17), the grain displacements and rotations are based on
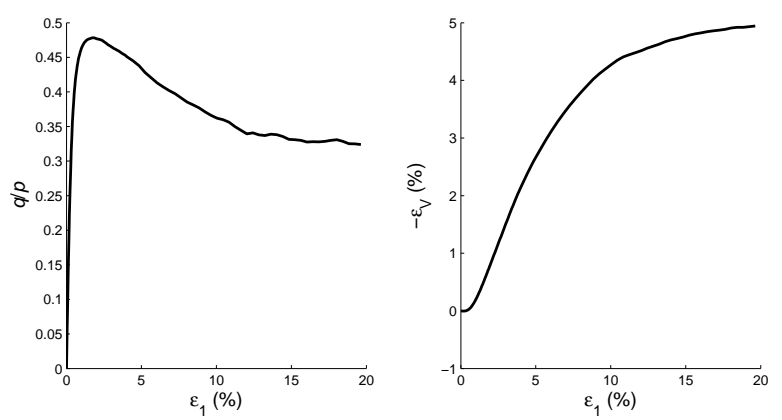

Fig. 5: Macroscopic response in a biaxial test employing periodic boundaries. Left: shear strength $q / p$; right: volumetric strain $\epsilon_{V}$. two states (or "snapshots") of the assembly (with corresponding grain positions and orientations), from which the grain displacements and rotations are determined. The tessellation is based on the "previous" snapshot. Note that the tessellation is only required to determine the loops. The displacements follow from the difference in positions of the grains, irrespective of the nature of the contacts (maintained, disrupted or created between the snapshots).

The micromechanical expression then provides the increment of the average displacement gradient of the assembly between these two states. The values for the components of the displacement gradient determined from the micromechanical expression, Eqs. (16) and (17), are visually indistinguishable from the macroscopic equivalent that is determined from the macroscopic deformation of the periodic box (and are therefore not shown separately in Figure 5). Quantitatively, at the final configuration the relative difference is less than $0.4 \%$ (the deviation being due to the incremental calculation of the total strains according to the micromechanical expression from the increments).

\subsection{Shear test}

As an additional verification of the developed micromechanical expression, a shear test is considered where periodic boundary conditions have again been employed. Here the shear deformation is imposed, while the vertical stress is kept constant. This is depicted schematically in Figure 6. The shear component of the deformation is characterised by the angle $\gamma$. The same initial assembly and the same contact model (and associated parameters) are used as for the DEM simulation of the biaxial test in Section 5.1. In the shear test the average rotation of the grains is not equal to zero, contrary to that in the biaxial test. Therefore, the shear test is more complex.

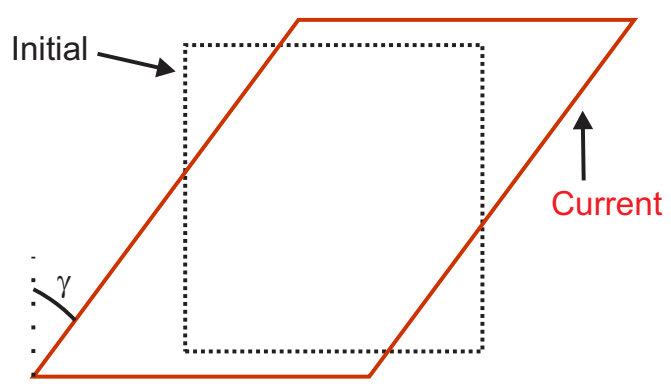

Fig. 6: Schematic of shear deformation. Shown are the initial periodic box and the current periodic box, as characterised by the angle $\gamma$ of the side of the periodic box with the vertical direction. 

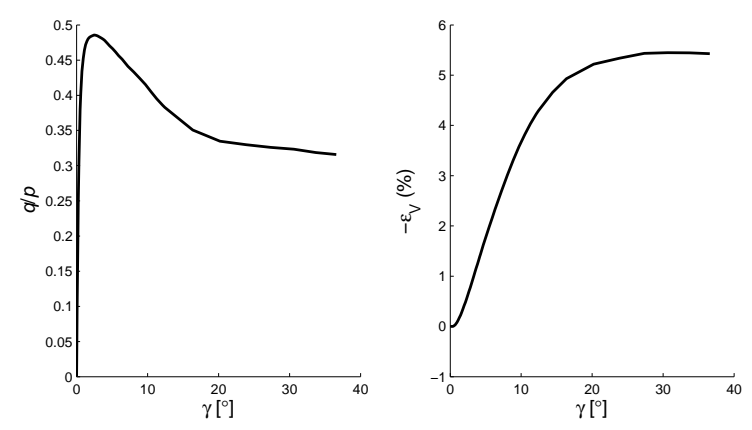

Fig. 7: Macroscopic response in a shear test employing periodic boundaries. Left: shear strength $q / p$; right: volumetric strain $\epsilon_{V}$.

The macroscopic response, in terms of shear strength $q / p$ and volumetric strain $\epsilon_{V}$ is shown in Figure 7. This is (expectedly) very similar to the response in the biaxial test (shown in Figure 5).

The average displacement gradients according to the micromechanical expression, Eqs. (16) and (17), and according to the macroscopic deformation of the periodic box agree very well (the components $\alpha_{12}$ and $\alpha_{21}$ according to the micromechanical expression are effectively zero, which is the macroscopic value). Quantitatively, at the final configuration the relative difference is less than $0.2 \%$ (the deviation again being due to the incremental calculation of the total strains according to the micromechanical expression from the increments.

\section{Shear band analysis}

The results of the analysis of the biaxial and the shear test have demonstrated that the developed micromechanical expression for the average displacement gradient tensor correctly reconstructs the overall, macroscopic deformation. Therefore, this expression will now be employed to analyse more complex deformation that involves a shear band.

For this case results from a DEM simulation are used that are slightly different from the DEM simulations described in Section 5. A biaxial test is again considered, but with flat, rigid, physical walls (instead of the periodic boundaries employed in the DEM simulations in Section 5). The number of grains in this simulation is 20150, which is smaller than that in the simulations reported in Section 5. The initial assembly has been created using a method similar to that described in Section 5. In the subsequent simulation, an interparticle friction coefficient $\mu=0.7$ and a stiffness ratio $k_{t} / k_{n}=0.42$ have been employed. The particle-wall friction coefficient equals zero. The initial solid fraction
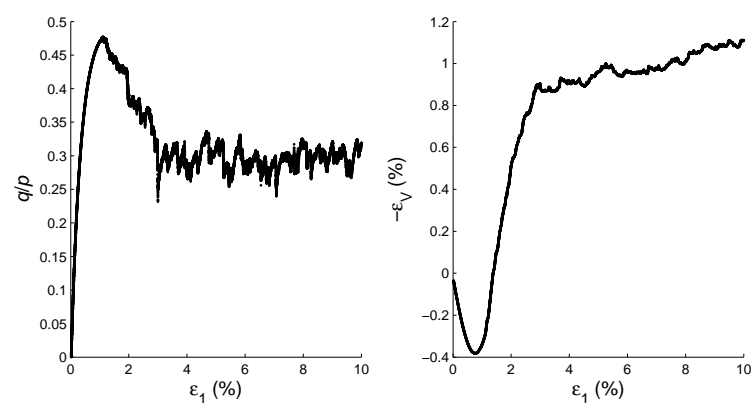

Fig. 8: Macroscopic response in a biaxial test employing physical walls. Left: shear strength $q / p$; right: volumetric strain $\epsilon_{V}$.

of the sample was 0.852 . Full details of this simulation are given in [22].

The macroscopic response, in terms of the shear strength $q / p$ and the volumetric strain $\epsilon_{V}$, is shown in Figure 8 . The shear strength $q / p$ shows a marked decrease following the peak shear-strength. Then a residual deviatoric stress plateau is reached. The volumetric strain shows the usual trends for dense specimens. After a first contactant stage, the specimen dilates, then shows a nearly isochoric behaviour. This behaviour occurs at the same time as the development of the deviatoric stress plateau. It corresponds to the so-called critical state known in soil mechanics: the material undergoes large shear strains under a constant deviatoric stress, without volume strain. In [22], the instability characteristics of the results of this simulation are analysed in detail, where it is shown that a shear band arises at the state of peak shear-strength.

Note that in the micromechanical formulation the outer boundary of the full system does not coincide with the physical walls, but instead is composed of the boundaries of the outer loops. Thus, in the present formulation the void cells adjacent to the physical walls are ignored.

In the following, the average displacement gradient tensor is investigated in Section 6.1. The spatial variation of the displacement gradient tensor, with an emphasis on the shear band region, is reported in Section 6.2 .

\subsection{Average displacement gradient}

Increments of the average displacement gradient of the whole system (i.e. the macroscopic value based on the deformation of the walls) and according to the micromechanical expression (based on Eqs. (16) and (17)) have been determined over the range of imposed strain, $\epsilon_{1}$. 


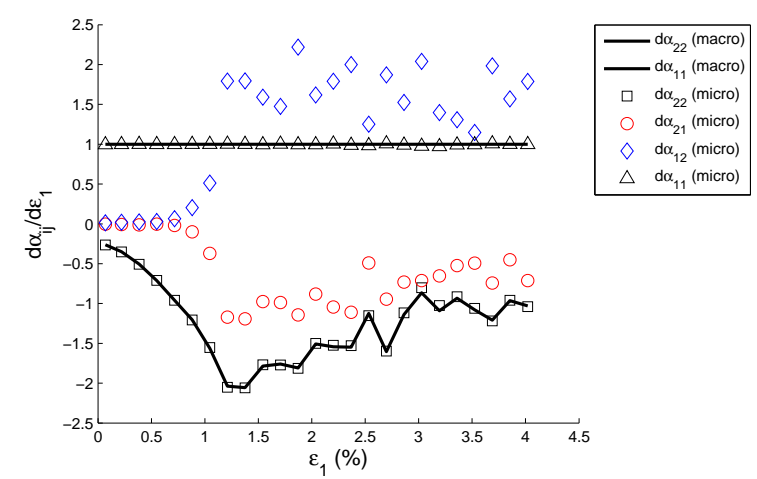

Fig. 9: Components of macroscopic and micromechanical displacement gradient tensors.

The results are shown in Figure 9. The diagonal components $d \alpha_{11}$ and $d \alpha_{22}$ of the increments of the macroscopic and the micromechanical displacement gradient agree very well over the whole range of imposed deformation. The off-diagonal components, $d \alpha_{12}$ and $d \alpha_{21}$, of the displacement gradient tensor are expected to be zero in biaxial tests (this is the value corresponding to the deformation of the walls, i.e. the macroscopic value). This is the case for the micromechanical expression for small imposed strain $\epsilon_{1}$ only. At larger imposed strain $\epsilon_{1}$, the off-diagonal components are not equal to zero. Hence, the solid walls enforce the motion of the system, but they allow for some shear and rotation of the assembly within the walls.

The origin of the discrepency between off-diagonal components of the increments of the macroscopic and the micromechanical displacement gradient tensors is investigated by replotting the previous data, with shear strength $q / p$ along the horizontal axis and $d \alpha_{12}$ along the vertical axis. Figure 10 shows that the off-diagonal component $d \alpha_{12}$ initially is small in the pre-peak/peak regime where the shear strength $q / p$ increases from nearly zero in the initial state to the state of peak shearstrength when the shear band is formed. In the postpeak regime (i.e. for strains larger than that at which the peak shear-strength occurs), there is a significant difference between the expected macroscopic value of zero of $d \alpha_{12}$ and that according to the micromechanical expression (see the upper set of data points in Figure $10)$.

For states of the assembly where the shear band is present, an investigation of the displacements of grains located near the walls demonstrated (data not shown) that the grain displacement field corresponds to that schematically indicated in Figure 11. This near-wall displacement field results in non-zero off-diagonal compo-

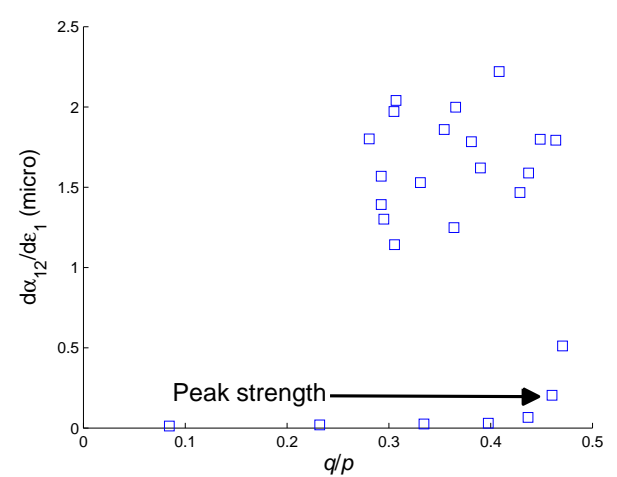

Fig. 10: Relation between shear strength $q / p$ and offdiagonal component of the increment of the average displacement gradient tensor, $d \alpha_{12}$, according to the micromechanical expression.

nents of the average displacement gradient tensor, according to Eq. (3).

To investigate the spatial homogeneity of the assembly, it is divided into three parts, as indicated in Figure 11. The location of the shear band (when it is present) has been estimated visually from the grain displacements. This corresponds to part B. The lower part is Part A and the upper part is Part C.

The components of the average displacement gradient tensors of these three parts have been determined,
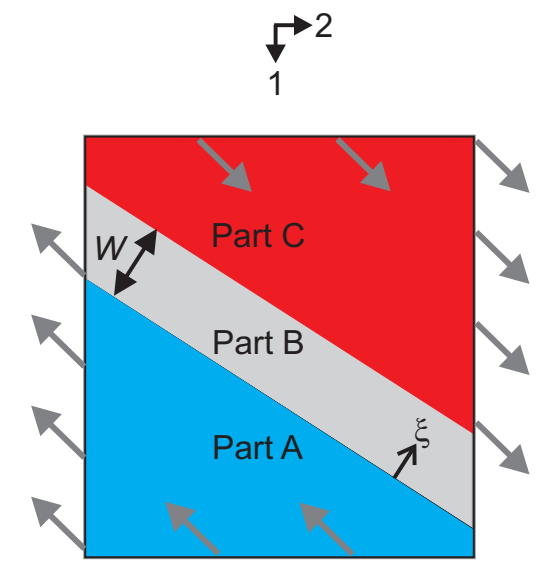

Fig. 11: Parts A, B and C of the assembly, where Part $\mathrm{B}$ corresponds to the location of the shear band, as estimated by visual observation. The width of the shear band is denoted by $W$ (this width is about 30 times the average grain diameter). A coordinate $\xi$ is indicated that represents the location inside the shear band; at the bottom of the shear band $\xi=0$, while $\xi=W$ at the top. The arrows schematically represent the displacements of grains that are located near the walls for states of the assembly where the shear band is present. 

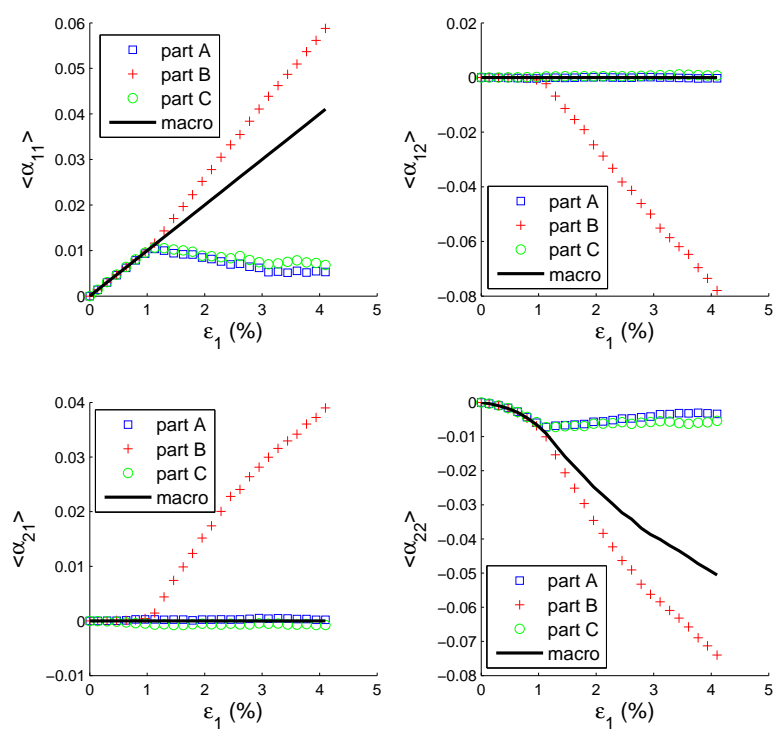

Fig. 12: Components of the average displacement gradient tensor of parts of the assembly These parts are indioated in Figure 12.

using the developed micromechanical expression, Eqs. (16) and (17). The results are shown in Figure 12, together with the macroscopic displacement gradient tensor as determined from the deformation of the bounding walls. Up to an imposed strain $\epsilon_{1}$ of $1 \%$, the displacement gradient in all three parts is the same and equal to the macroscopic displacement gradient. This is characteristic of homogeneous deformation. At strains $\epsilon_{1}$ larger than $1 \%$, deformation mainly occurs within the shear band. The upper and lower parts $\mathrm{A}$ and $\mathrm{C}$ behave as nearly-rigid bodies. Note also that the off-diagonal components $d \alpha_{12}$ and $\mathrm{d} \alpha_{21}$ are effectively zero outside of the shear band. The micromechanical deformation measures also demonstrate a clear strain localisation.

\subsection{Spatial variation of displacement gradient}

To assess the spatial variability of the deformation of all the loops, the increment of the volumetric strain $\left\langle d \epsilon_{V}\right\rangle_{S_{L}}$ is shown in Figure 13 in normalised form. This Figure shows regions with small strains, i.e. regions that behave as nearly-rigid bodies (such as Parts A and $\mathrm{C}$ in Figure 11). The deformation is localised in the shear band where the deformation is large. Notable is the significant heterogeneity of the deformation in the shear band and the adjacent regions. Smaller patches with significant deformation are also present outside of the shear band.

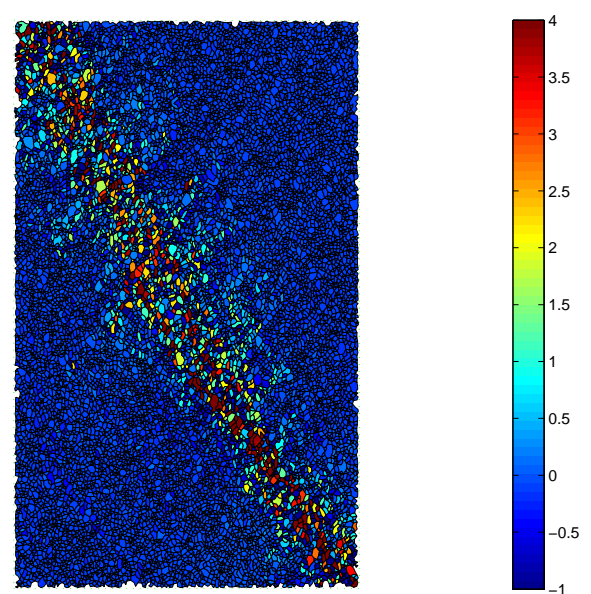

Fig. 13: Increment of the average volumetric strain of loops, based on two states ("snapshots") near the state with peak shear strength. Shown are normalised volumetric strains, i.e. the ratio between actual volumetric strain relative to the mean volumetric strain over the standard deviation of the volumetric strains.

Figure 13 shows that there is considerable heterogeneity in the average displacement gradients of the loops. To investigate whether patterns exist for the variation of the displacement gradient tensor inside the shear band, the average displacement gradient tensors of the loops are averaged in the direction transverse to the shear band. As a result, the spatial dependence of

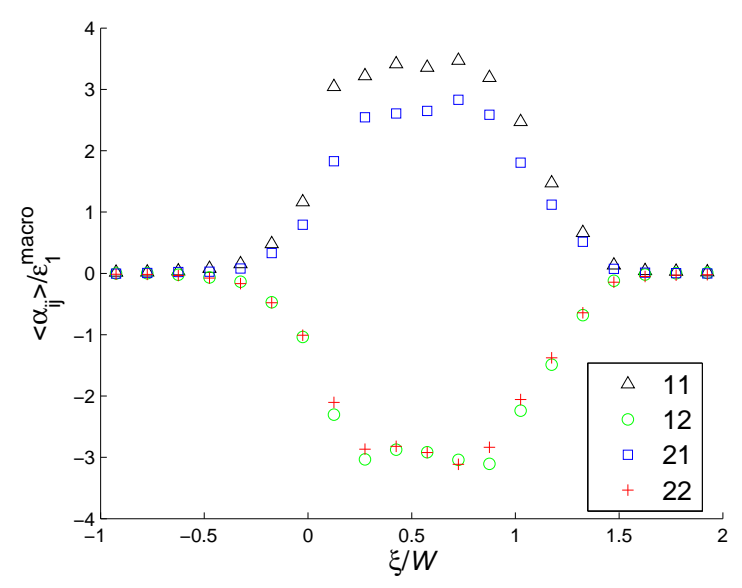

Fig. 14: Profile over the shear band of the transverselyaveraged (total) displacement gradient tensor at the final state; $\xi$ is a coordinate perpendicular to the shear band, see Figure 11 and $W$ is the width of the shear band, based on visual observation. 
the transversely-averaged displacement gradient $\bar{\alpha}_{i j}(\xi)$ is obtained, where $\xi$ is the coordinate perpendicular to the shear band; $\xi=0$ corresponds to the bottom of the shear band, while $\xi=W$ corresponds to the top (see also Figure 11). The results for $\bar{\alpha}_{i j}(\xi)$ are shown in Figure 14.

Since $d \alpha_{12} \neq d \alpha_{21}$, an average rotation is present inside the shear band. Furthermore, the material dilates inside the shear band. At the edges of the shear band ( $\xi=0$ and $\xi=W$ ), as determined visually, the displacement gradient tensor does not equal zero. Hence, the shear band is thicker than expected based on the visual estimate.

When the contribution of the grain rotations to the average displacement gradient tensor in Eq. (16) is evaluated separately, it is found that its magnitude is much smaller (only a few percent) than the magnitude of the grain displacement contribution. This leads to the (negative) conclusion that the influence of the grain rotations on the average displacement gradient is negligible for dense assemblies, even in cases (such as the shear band considered here) where large gradients in grain displacements are present.

\section{Conclusions}

A surface-additive micromechanical formulation for the average displacement gradient has been proposed that forms a synthesis of previous work by incorporating the contribution of grain rotations and by expressing it in terms involving the relative displacements at intergrain contacts.

The resulting expression has been verified by comparing its results with the macroscopic deformation, as determined from the boundary displacements. For a biaxial test and a shear test, both employing periodic boundary conditions, good agreement has been found.

Using the developed micromechanical formulation, the deformation in a complex case involving a shear band has been analysed. Initially, the microscopic and macroscopic average displacement gradients are equal. At the state of peak shear-strength, deviations arise for the off-diagonal components of the displacement gradient tensor. This means that the assembly undergoes some shear and rotation inside the physical bounding walls.

The spatial homogeneity of the deformation has been studied by using the developed micromechanical expression for the deformation gradient tensor to different parts of the assembly. Up to the state of peak shearstrength, the deformation is homogeneous, but for larger imposed strains, clear localisation of the deformation is observed with large deformation inside the shear band, while the regions oustide of the shear band behave as semi-rigid bodies.

The average displacement gradient tensors of the loops show strong spatial heterogeneity. Patches of loops with large deformation are also observed outside of the shear band.

As a step towards detailed investigations of the kinematics inside shear bands, the average displacement gradient has been transversely averaged. The resulting profiles for the variation of the displacement gradient perpendicular to the shear band show clear patterns. The detailed investigation of these profiles is hampered by the limited size of the considered assembly.

The contribution of rotations to the average displacement gradient has been shown to be negligible for the dense assemblies considered here, even in a case of system with a shear band where large gradients in the grain displacements exist.

For future studies of deformation inside shear bands it is recommended to employ (much) larger assemblies in order to obtain (more) reliable results. The current study does not provide for an expression for the average (Cosserat) rotation. The formulation of such an expression will be reported in future work.

\section{Acknowledgements}

The authors acknowledge data shown in Section 6 that was kindly provided by N. Hadda (previously with Irstea, France; currently with the Department of Civil Engineering of the University of Calgary, Alberta, Canada). NK acknowledges hospitality at Université de La Rochelle.

\section{Disclosures}

The authors declare that there are no undisclosed conflicts of interest (both personal and institutional) regarding specific financial interests that are relevant to the work conducted or reported in this manuscript.

\section{References}

1. Aboudi, J., Arnold, S.M., Bednarcyk, B.A. (2013) Micromechanics of composite materials. Elsevier, Amsterdam, the Netherlands.

2. Ancey, C. (2002) Dry granular flows down an inclined channel: experimental investigations on the frictionalcollisional regime. Physical Review E 65: 011304.

3. Aranson, I.S. \& Tsimring, L.S. (2001) Continuum theory of partially fluidized granular flows. Physical Review E 64: 020301.

4. Bagi, K. (1996) Stress and strain in granular assemblies. Mechanics of Materials 22: 165-177. 
5. Bagi, K. (2006) Analysis of microstructural strain tensors for granular assemblies. International Journal of Solids and Structures 43: 3166-3184.

6. Bonelli, S. \& Millet, O. \& Nicot, F. \& Rahmoun, J. \& de Saxcé G. (2012) On the definition of an average strain tensor for two-dimensional granular material assemblies, International Journal of Solids and Structures 49: 947958.

7. Cambou, B., Chaze, M., Dedecker, F., (2000) Change of scale in granular materials. Eur. J. Mech. A/Solids 19: 999-1014.

8. Cambou, B., Jean, M., (2001) Micromécanique des milieux granulaires. Hermes, Paris.

9. Chang, C.S., Gao, J. (1996) Kinematic and static hypotheses for constitutive modelling of granulates considering particle rotation. Acta Mechanica 115: 213-229.

10. Cundall, P.A., Strack, O.D.L. (1979) A discrete numerical model for granular assemblies. Géotechnique 9: 4765.

11. Dantu P. (1957) Contribution à l'étude mécanique et géométrique des milieux pulvérulents, Proc. 4th Int. Conf. Soils Mech. Found. Eng., London, pp. 144-148.

12. Dedecker, F., Chaze, M., Dubujet, Ph., Cambou, B. (2000) Specific features of strain in granular materials. Mech. Cohes.-Frict. Mater. 5: 173-193.

13. de Saxcé, G., Fortin, J., Millet, O. (2004) About the Numerical Simulation of the Dynamics of Granular Media and the Definition of the Mean Stress Tensor, Mechanics of Materials, (36) pp. 1175-1184.

14. Durán, O. \& Kruyt, N.P. \& Luding, S. (2010) Micro-mechanical analysis of deformation characteristics of three-dimensional granular materials. International Journal of Solids and Structures 47 2234-2245.

15. Durán, O. \& Kruyt, N.P. \& Luding, S. (2010) Analysis of three-dimensional micro-mechanical strain formulations for granular materials: evaluation of accuracy. International Journal of Solids and Structures 47 251-260.

16. Eringen, A.C.: Microcontinuum field theories I: Foundations and solids, Springer, New York, USA (1999).

17. de Gennes, P.G. (1998) Reflections on the mechanics of granular matter. Physica A 261, pp. 267-293.

18. Fortin, J., Millet, O., De Saxcé, G. (2002) Mean stress in a granular medium in dynamics, Mechanics Research Communications 29 (4), pp. 235-240.

19. Fortin, J., Millet, O., De Saxcé, G. (2003) Construction of an averaged stress tensor for a granular medium, European Journal of Mechanics, A/Solids 22 (4), pp. 567-582.

20. J. Fortin, O. Millet, G. de Saxcé (2005) Numerical Simulation of Granular Materials by an improved Discrete Element Method, Int. J. for Num. Methods in Engineering, (62) pp. 639-663.

21. Goldhirsch, I., Goldenberg, C. (2005) Continuum mechanics for small systems and fine resolutions. In: Rieth, M., Schommers, W. (Eds.), Handbook of Theoretical and Computational Nanotechnology. American Scientific Publishers, Stevenson Ranch, CA, USA, pp. 1-58.

22. Hadda, N. (2012) Aspects micromécaniques de la rupture dans les milieux granulaires (in French) [Micromechanical aspects of failure in granular materials]. Ph.D. Thesis Université de Grenoble, Grenoble, France.

23. Horne, M.R. (1965) The behaviour of an assembly of rotund, rigid, cohesionless particles, I and II. Proceedings of the Royal Society of London A 286: 62-97.

24. Kruyt, N.P., Rothenburg, L. (1996) Micromechanical definition of the strain tensor for granular materials. Journal of Applied Mechanics (Transactions of the ASME) 63: 706-711.
25. Kruyt, N.P. (2003) Statics and kinematics of discrete Cosserat-type granular materials, International Journal of Solids and Structures 40: 511-534.

26. Kuhn, M.R. (2004) A boundary integral for gradient averaging in two dimensions: application to polygonal regions in granular materials. International Journal for Numerical Methods in Engineering 59: 559-576.

27. Liao, C.-L., Chang, T.-P., and Young, D.-H. (1997) Stress-strain relationship for granular materials based on the hypothesis of best fit. International Journal of Solids and Structures 34: 4087-4100.

28. Nicot, F., Darve, F. (2005) A multi-scale approach to granular materials. Mechanics of Materials 37: 980-1006.

29. Oda, M., and Konishi, J. (1974) Microscopic deformation mechanism of granular material in simple shear. Soils and Foundations 14: 15-32.

30. O'Sullivan, C., Bray, J.D., and Li, S.F. (2003) A new approach for calculating strain for particulate media, International Journal for Numerical and Analytical Methods in Geomechanics 27: 859-877.

31. Pouliquen, O., Forterre, Y., and Ledizes, S. (2001) Dense granular flows down incline as a self-activated process. Advances in Complex Systems 4: 441-450.

32. Radjai, F., Wolf, D., Jean, M., and Moreau, J.J. (1998) Bimodal character of stress transmission in granular packing. Physical Review Letters 80: 61-64.

33. Radjai, F., Roux, S., and Moreau, J.J. (1999) Contact forces in a granular packing. Chaos 9: 544-550.

34. Rajchenbach, J. (2000) Granular flows. Advances in Physics 49: 229-256.

35. Tordesillas, A., Muthuswamy, M. (2009) On the modeling of confined buckling of force chains. Journal of the Mechanics and Physics of Solids 57: 706-727.

36. Tordesillas, A., Walker, D.M., and Lin, Q. (2010) Force cycles and force chains. Physical Review E 81: 011302.

37. Tordesillas, A., Pucilowski, S., Sibille, L., Nicot, F., and Darve, F. (2012) Multiscale characterization of diffuse granular failure. Philosophical Magazine 92: 4547-4587. 